\title{
Male Breast Cancer: a 24 Year Experience of a Tertiary Care Hospital in Pakistan
}

\author{
Omer Jamy ${ }^{1 *}$, Ammar Rafiq ${ }^{2}$, Altaf Laghari², Tabish Chawla ${ }^{2}$
}

\begin{abstract}
Background: Male breast cancer accounts for less than $1 \%$ of all cancers found in men. It usually presents at a later age and stage as compared to female breast cancer. Treatment strategies are extrapolated from the management of female breast cancer. Our study here looked at 18 patients diagnosed with and treated for male breast cancer at The Aga Khan University Hospital in Pakistan. We compared our findings with the existing data from Asian and Western countries. Materials and Methods: A retrospective study was conducted looking at patients with male breast cancer between January 1986 and December 2009. Patient and disease characteristics were analyzed and 5 year overall survival was calculated using Microsoft Excel and SPSS. Results: The average age at diagnosis was 52 years (38-67 years). Twelve $(66.7 \%)$ patients had axillary lymphadenopathy. Stage II disease was the most common stage at presentation ( 9 patients, $50 \%$ ). Infiltrating ductal carcinoma was seen in 16 patients $(\mathbf{8 8 . 8 \%})$. Seven lesions were positive for both estrogen and progesterone receptors. Sixteen patients had surgery in the form of either modified radical mastectomy or radical mastectomy. Radiation was used in 7 patients in an adjuvant setting. The five year overall survival for stage I, II, III and IV disease was $100 \% v s$ $78 \%$ vs $50 \%$ vs $0 \%(\mathrm{p}<0.05)$. Five year overall survival was $61 \%$. None of the other prognostic factors were statistically significant. Median follow up was 15 months (3-202 months). Conclusions: Male breast cancer may be on a slow rise but is still an uncommon disease. Tumor stage and lymph node status are important prognostic markers. Public awareness and screening may help in detecting the disease at an earlier stage. Prospective trials are needed to improve the management of this disease.
\end{abstract}

Keywords: Male breast cancer - female breast cancer - overall survival - prognostic factors - Pakistan

Asian Pac J Cancer Prev, 16 (4), 1559-1563

\section{Introduction}

Male breast cancer (MBC) accounts for less than $1 \%$ of all cancers in men as well as all breast carcinomas. Given the rarity of the disease, most of the information regarding its characteristics, management and outcome is based on either retrospective studies of a small number of patients or extrapolation of the results of female breast cancer (FBC). (Giordano et al., 2004; Nahleh et al., 2007; Sedighi et al., 2013) Recently published data in the United States has shown a significant increase in the incidence of MBC whereas a steady low rate has been observed in European studies. (Kaushik et al., 2014) There is still a paucity of randomized controlled trials and treatment for MBC is based on treatment for FBC.

The general variation of MBC across the globe is similar to FBC with higher rates in North America and Europe and lower rates in Asia. (Chikaraddi et al., 2012; Kaushik et al., 2014) There have been studies from Pakistan describing the histopathology of MBC in different regions. Studies describing the clinical aspect of $\mathrm{MBC}$ in the Pakistani population are limited. We looked at the data of 18 patients with MBC at our institute and analyzed their clinicopathological features. We also compared our results to Western literature to see how MBC behaved in different populations.

\section{Materials and Methods}

This retrospective review was carried out at The Aga Khan University Hospital (AKUH) in Karachi, Pakistan. Approval was obtained from the ethics committee at AKUH. Files with ICD code 175 (Malignant neoplasm of male breast) were retrieved from patient medical records. A total of 19 patients with MBC were seen at our institute between January 1986 and December 2009. One patient with missing records was excluded from the study. Data from 18 files were reviewed and analyzed.

The variables studied were age at diagnosis, comorbid conditions, presenting signs and symptoms (breast lump, erythema, nipple discharge and axillary lymphadenopathy), family history of breast cancer, smoking history, diagnostic modality, histopathology including hormonal work up, TNM staging, treatment 
(surgery, chemotherapy and radiotherapy), and outcome (follow up, recurrence and five year survival).

Information on chemotherapy administration was obtained from patient records. Specific details of chemotherapy, however, were not available during file review. Genetic testing was not offered at our institute.

Data was tabulated using Microsoft Excel 2010 and analyzed using SPSS bio-statistical software (version 19.0). Continuous variables were analyzed as means and categorical variables were analyzed as percentages. Overall survival (OS) was estimated with the KaplanMeier product-limit method. A $p$ value of $<0.05$ was considered significant.

\section{Results}

The average age at diagnosis was 52 years (range 38-67 years). All patients presented with a breast lump. Twelve $(66.6 \%)$ patients had right sided involvement and $6(33.3 \%)$ patients had left sided involvement. Axillary lymph nodes were palpable in $12(66.6 \%)$ patients. Other presenting symptoms included breast erythema $(55.5 \%)$ and nipple discharge (22.2\%).

On questioning, $5(27.7 \%)$ patients had a positive family history for breast cancer. Seven (38.9\%) patients had hypertension, diabetes or both as co-morbid conditions. Two patients had chronic lung disease. Nine (50\%) patients were smokers. None of the patients had a history of Klinefelter's syndrome, gynecomastia, radiation exposure or any underlying liver disorder.

Diagnostic modalities included fine needle aspirate cytology in 12(66.6\%) patients and trucut biopsy in 6 $(33.3 \%)$. These procedures were performed at AKUH.

Histopathological reports confirmed $16(88.8 \%)$ of the tumors as Infiltrating Ductal Carcinoma (IDC), 1 (5.5\%) as infiltrating adenocarcinoma and $1(5.5 \%)$ as intra-cystic papillary carcinoma. Amongst these cases, 4 (22.2\%) had stage I, $9(50 \%)$ had stage II, $2(11.1 \%)$ had stage III and $3(16.7 \%)$ had stage IV disease. Hormonal studies were conducted in 15 patients out of whom 7 were both
Estrogen receptor (ER) and Progesterone receptor (PR) positive, 3 were only ER positive and 5 were negative for both. HER 2 and genetic testing were not done at our institute. None of the patients had distant metastasis at initial presentation. Disease characteristics are listed in Table 1.

Surgical intervention was offered to all the patients. Twelve $(66.7 \%)$ opted for modified radical mastectomy (MRM) and 4 (22.2\%) for radical mastectomy (RM). Two (11.1\%) patients refused surgical intervention. Neoadjuvant chemotherapy was given to $6(33.3 \%)$ patients whereas $8(44.4 \%)$ patients received adjuvant chemotherapy. Two patients did not receive chemotherapy

Table 1. Patient and Disease Characteristics

\begin{tabular}{|c|c|c|}
\hline Characteristic & & ale No. $(\%)$ \\
\hline \multirow[t]{3}{*}{ Histopathology } & Invasive Ductal & $16(88.8)$ \\
\hline & Infiltrating Adeno & 1 (5.6) \\
\hline & Intra Cystic Papillary & $1 \quad(5.6)$ \\
\hline \multirow[t]{4}{*}{ Cancer Stage } & 1 & $4(22.20$ \\
\hline & 2 & $9(50)$ \\
\hline & 3 & $2(11.1)$ \\
\hline & 4 & $3(16.7)$ \\
\hline \multirow[t]{2}{*}{ Lymph Nodes present } & Yes & $12(66.7)$ \\
\hline & No & $6(33.3)$ \\
\hline \multirow[t]{2}{*}{ Estrogen Receptor * } & Positive & $10(66.7)$ \\
\hline & Negative & $5(33.3)$ \\
\hline \multirow[t]{2}{*}{ Progesteron Receptor * } & Positive & $7(46.7)$ \\
\hline & Negative & $8(53.3)$ \\
\hline \multirow[t]{3}{*}{ Laterality } & Right & $12(66.7)$ \\
\hline & Left & $6(33.3)$ \\
\hline & Both & $0 \quad(0.0)$ \\
\hline \multirow[t]{2}{*}{ Hormonal Therapy } & Tamoxifen & $10(55.6)$ \\
\hline & Not Given & $8(44.4)$ \\
\hline \multirow[t]{4}{*}{ Chemotherapy } & Neoadjuvent & $6(33.3)$ \\
\hline & Adjuvent & $8(44.4)$ \\
\hline & Not Given & $2(11.1)$ \\
\hline & Refused & $2(11.1)$ \\
\hline \multirow[t]{2}{*}{ Radiotherapy } & Yes & $7(38.9)$ \\
\hline & No & $11(61.1)$ \\
\hline
\end{tabular}

*Both ER and PR were done on 15 patients out of 18 due to the unavailabilty of data

Table 2. Prognostic Factors of Survival in Male Breast Cancer Patients

\begin{tabular}{|c|c|c|c|c|c|}
\hline & & $\begin{array}{l}\text { Percentage of patients } \\
\text { with disease }\end{array}$ & $\begin{array}{l}5 \text { Year Survival } \\
\text { Percentage }\end{array}$ & Log Rank test & $\mathrm{p}$ value \\
\hline \multirow[t]{3}{*}{ Histopathology } & Invasive Ductal & $88.8 \%$ & $62.5 \%$ & 2.914 & 0.2330 \\
\hline & Infiltrating Adeno & $5.6 \%$ & $0.0 \%$ & & \\
\hline & Intra Cystic Papillary & $5.6 \%$ & $100.0 \%$ & & \\
\hline \multirow[t]{4}{*}{ Cancer Stage } & 1 & $22.2 \%$ & $100.0 \%$ & 18.748 & $0.0003^{2}$ \\
\hline & 2 & $50.0 \%$ & $77.8 \%$ & & \\
\hline & 3 & $11.1 \%$ & $50.0 \%$ & & \\
\hline & 4 & $16.7 \%$ & $0.0 \%$ & & \\
\hline \multirow[t]{2}{*}{ Estrogen Receptor ${ }^{1}$} & Positive & $66.7 \%$ & $70.0 \%$ & 0.432 & 0.5110 \\
\hline & Negative & $33.3 \%$ & $80.0 \%$ & & \\
\hline \multirow[t]{2}{*}{ Progesteron Receptor ${ }^{1}$} & Positive & $46.7 \%$ & $71.0 \%$ & 0.170 & 0.6800 \\
\hline & Negative & $53.3 \%$ & $62.5 \%$ & & \\
\hline \multirow[t]{2}{*}{ Lymph Nodes present } & Yes & $66.7 \%$ & $58.3 \%$ & 0.290 & 0.5910 \\
\hline & No & $33.3 \%$ & $66.7 \%$ & & \\
\hline \multirow[t]{2}{*}{ Smoking } & Yes & $50.0 \%$ & $66.7 \%$ & 1.397 & 0.2370 \\
\hline & No & $50.0 \%$ & $44.4 \%$ & & \\
\hline \multirow[t]{2}{*}{ Family History of Cancer } & Yes & $27.8 \%$ & $60.0 \%$ & 0.012 & 0.9140 \\
\hline & No & $72.2 \%$ & $53.8 \%$ & & \\
\hline
\end{tabular}

${ }^{* 1}$ Both ER and PR were done on 15 patients out of 18 due to the unavailabilty of data; ${ }^{2} \mathrm{p}<0.05$; statistically significant 


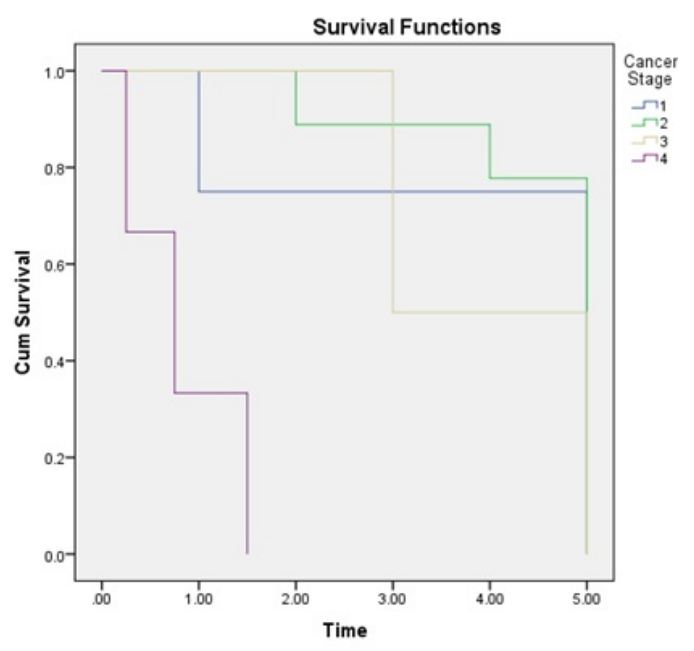

Figure 1. Kaplan-Meier Survival Curve Based on Stage of Cancer

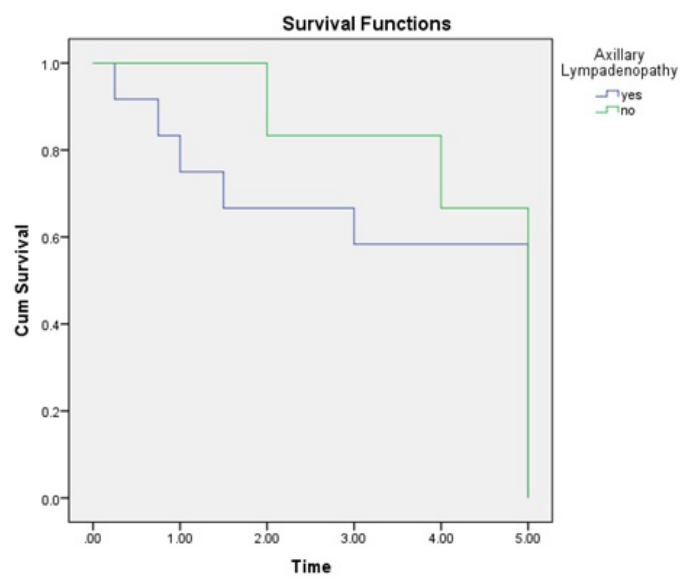

Figure 2. Kaplan-Meier Survival Curve Based on Lymph Node Status

and 2 refused to take it. The medical records did not contain data regarding the details of chemotherapy. Seven $(38.9 \%)$ received adjuvant radiotherapy. Tamoxifen was given as hormonal therapy to all with positive receptors.

Follow up ranged from 3 to 202 months with the median being 15 months. On follow up, six patients had distant metastasis with the bone involved in 4 cases and the liver and brain in 1 each. The patient with liver metastasis passed away in 9 months whereas the patient with brain metastasis passed away in 3 . Two patients with bone metastasis passed away within two years. Two patients had local recurrence within a year. Both were treated with chemotherapy.

Five year overall survival (OS) varied by stage. Patients with stage I disease had a 5 year OS of $100 \%$ compared to $77.8 \%$ with stage II, $50 \%$ with stage III and $0.0 \%$ with stage IV. Prognostic factors of survival in MBC are given in detail in table 2. Five year OS was $61.1 \%$. Our results showed that stage dependant 5 year OS was the only statistically significant result $(\mathrm{p}<0.05)$. Figure 1 and Figure 2 show survival curves of tumor stage and axillary lymph node status respectively.

\section{Discussion}

Even though the incidence of $\mathrm{MBC}$ is becoming similar to that of FBC, it is still an uncommon disease. Because of the rarity of $\mathrm{MBC}$, information regarding patient and disease characteristics is limited. (Nahleh et al., 2007; Anderson et al., 2010)

MBC most commonly targets men in the 6th and 7 th decade of life. The age at diagnosis for men is 5 to 10 years higher than that for women. Along with a higher age, men also present with more advanced disease. This can be attributed to factors such as lack of screening mammography and public awareness. (Giordano et al., 2004; Miao et al., 2011; Shah et al., 2012; Kaushik et al., 2014) The average age at diagnosis in our population was 52 years with 7 of our patients falling in the age group 50 to 55 . This was 5 years younger than the age of diagnosis of women with FBC in Pakistan. (Tasneem et al., 2011) More than half of our patients had stage II disease with stage I being the second most common. Our average age at diagnosis was slightly younger than that mentioned in Western literature but was similar to reports from India, Egypt and Iran. (Shah et al., 2012; Sedighi et al., 2013; Soliman et al., 2014) Hyperestrogenism caused by liver failure secondary to infectious etiologies has been considered as a cause of relatively higher rates of MBC in developing regions of the world. (Kaushik et al.,2014) This can be one possible cause of a younger age at diagnosis in these studies. Genetic differences may be another reason but needs to be explored further.

Most men with breast cancer present with a breast lump. Nipple discharge, ulceration, retraction and localized pain are some other common presenting features. Axillary lymphadenopathy was also a common finding in $40-55 \%$ of the patients. Lymph node status has been implicated as an important prognostic marker of MBC. Lymph node positive MBC carried a poor prognosis as compared to node negative MBC. (Giordano et al., 2004; Zygogianni et al., 2012; Selcukbiricik et al., 2013) In our study, the 5 year OS for lymph node negative and positive patients was $66.7 \%$ and $58.3 \%$ respectively. However, this result was not statistically significant $(\mathrm{p}=0.591)$.

Mutations in BRCA 2 mainly and also BRCA 1 have been known to increase the risk of MBC. However, these genetic mutations only account for a small proportion of $\mathrm{MBC}$ occurrence. Studies have shown that a first degree relative with a history of breast cancer increased the risk of MBC. Klinefelter's syndrome has been associated with a 50-fold increase in risk of MBC. Obesity, physical inactivity, liver cirrhosis, hyperthyroidism, gallstones and diabetes have also been linked to an increased risk of MBC. An association between smoking and MBC has been studied but no convincing trend has been identified. As with FBC, there is an absence of a strong effect of smoking on MBC. (Jamal et al., 2006; Brinton et al., 2008; Zygogianni et al., 2012; Kaushik et al., 2014) The 5 year OS for smokers in our study was $66.7 \%$ which was higher than the $44.4 \%$ in non-smokers. This result was not statistically significant though $(\mathrm{p}=0.237)$. Six patients in our study population had a family history positive for breast cancer. The 5 year OS for those patients was $60.8 \%$ compared to $53.8 \%$ for patients without a positive family history $(\mathrm{p}=0.914)$. However, genetic testing was not available to check for commonly occurring mutations 
in those cases. None of our patients had a history of Klinefelter's syndrome or any underlying liver disease.

IDC is the predominant histological type of $\mathrm{MBC}$ in both the Western and Asian population. (Giordano et al., 2004; Jamal et al., 2009; Sipetic-Grujicic et al., 2014) IDC is also the most frequent subtype in FBC. Papillary carcinoma is the second most frequent subtype in MBC. This is in contrast to invasive lobular carcinoma which is the second most common subtype in FBC. Lobular carcinoma is thought to be exclusive to FBC. However, literature has shown that exposure to estrogen can cause terminal lobules to develop in the male breast. The occurrence of lobular carcinoma in $\mathrm{MBC}$ is still rare. (Giordano et al., 2004; Chikaraddi et al., 2012; Zhou et al., 2014) Most of our patients had IDC and we did not see any cases of lobular carcinoma. Men are more likely to have ER and PR positive breast cancer (65-92\%). An improved OS for ER/PR positive MBC has been shown in the univariate analysis. However, in the multivariate analysis adjusted for tumor stage and lymph node status, the difference in OS was not significant. (Giordano et al., 2004; Sedighi et al., 2013; Selcukbiricik et al., 2013; Kaushik et al., 2014) In our population, $66.7 \%$ patients were ER positive and $46.7 \%$ were PR positive. All were treated with Tamoxifen. The 5 year OS for ER positive versus ER negative MBC was $70 \%$ and $80 \%$ respectively. This result was not statistically significant $(p=0.511)$. Five year OS for PR positive versus PR negative MBC was also not significant $(71.0 \%$ vs $62.5 \%, \mathrm{p}=0.680)$.

Various results have been published in regards to the most common stage of disease at initial presentation. Western literature has reported a more frequent occurrence of stage I and stage II disease. (Giordano et al., 2004; Nahleh et al., 2007) Literature from Asian countries has shown that stage III and stage IV disease occurs more frequently in their population. (Tasneem et al., 2011; Chikaraddi et al., 2012) Duration of symptoms before presentation is 1-8 months in the Western population and 12-15 months in the Asian and African population. (Kaushik et al., 2014) This can be one explanation for the difference in stage at presentation between the two populations. Social factors such as insurance status, access to primary health care and marital status can also influence early detection of MBC. Studies regarding these factors are lacking and can help us in understanding this difference. Stage II disease was the most common (50\%) stage in our population followed by stage I $(22.1 \%)$. This was inconsistent with the already existing South Asian data. One reason for this finding could be our small sample size. Another speculation for this finding can be that AKUH is a private hospital catering to mainly upper middle class population. Majority of the population of Pakistan cannot afford the costs incurred at this hospital and seek medical attention from public hospitals only when absolutely necessary. Based on this study, our sample may not be an actual representation of a developing country's population. However, Pakistan also ranks amongst the top nations when it comes to philanthropy and people from a low socioeconomic background are also able to get treatment at renowned institutes. (Kassim-Lakha and Bennett, 2013) Results from other studies on MBC in Pakistan have shown an increased frequency of late stage disease and therefore this speculation cannot be laid to rest without conducting further research looking into the social barriers of developing countries. (Tasneem et al., 2011)

The main principles of treating $\mathrm{MBC}$ have been extrapolated from the treatment of FBC. Surgery is the mainstay of treatment. RM has been replaced by MRM after retrospective studies suggested the equivalence of both procedures. Extensive chest involvement may be the only exception where RM is preferred. (Chikaraddi et al., 2012; Zygogianni et al., 2012) Neoadjuvant treatment depends upon hormonal status of the tumor and also on the inoperable nature of the tumor. Whereas women have the incentive of having breast conserving surgery with neoadjuvant treatment, men do not. Despite the dearth of randomized trials, adjuvant therapy has proven to improve the outcome of MBC. (Zygogianni et al., 2012; Sipetic-Grujicic et al., 2014) One prospective study used Cyclophosphamide, Methotrexate and Fluorouracil (CMF) to show a 20 year survival of $42 \%$ in 31 patients after 12 cycles. MD Anderson reported Adriamycin based chemotherapy to have a reduced risk of death as compared to CMF. (Shah et al., 2012; Zygogianni et al., 2012) Definite data on the treatment of MBC needs to be proven with prospective trials. Adjuvant hormonal therapy with Tamoxifen is given to ER and PR positive patients. The indication for radiation is the same as that for FBC and it has shown to decrease the chances of recurrence. However, it has little effect on the overall survival rate. (Shah et al., 2012; Zygogianni et al., 2012) MRM was the popular choice amongst our patients as well. One of the limitations of our study was the lack of chemotherapy detail available. Due to this reason a detail analysis of chemotherapy was not done. Seven patients received adjuvant radiotherapy. None of these patients had stage I disease. The 5 year OS for patients with $\mathrm{MBC}$ receiving radiation was $71 \%$. This result was not significant though. Tamoxifen was given to all patients positive for ER and PR.

Axillary lymph node status and tumor stage are the two most important prognostic factors for MBC. Omitting lymph node dissection has shown to have a worse outcome in MBC. (Chikaraddi et al., 2012; Kaushik et al., 2014) Giordano et al reported 5 year OS rates of $78 \%$ for stage I, $67 \%$ for stage II, $40 \%$ for stage III and $19 \%$ for stage IV disease. (Giordano et al., 2004) Our study had a $0 \% 5$ year OS for stage IV disease compared to $50 \%$ for stage III and $77.8 \%$ for stage II disease. Stage I disease had a $100 \% 5$ year OS. Even though this result was significant, our sample size was limited. The 5 year OS for MBC has ranged from $36 \%$ to $89 \%$ as per different studies. This range can be explained by variable stage of disease in different studies, different study populations and different treatment protocols used. (Kaushik et al., 2014) Most Western studies have reported a 5 year OS of greater than $60 \%$ whereas results of Asian and African countries have shown a 5 year OS ranging from 40-60\%. (Giordano et al., 2004; Miao et al., 2011; Shah et al., 2012; Sedighi et al., 2013) Our 5 year OS was $61.1 \%$ which was consistent with most of the South Asian studies as well as Western studies.

$\mathrm{MBC}$ is thought to have a worse outcome as compared to FBC. Reason for this include an older age at diagnosis, 
advanced stage at diagnosis and a lack of standard treatment for loco-regional disease. (Nahleh et al., 2007) However, Miao et al adjusted for the above mentioned factors and actually found $\mathrm{MBC}$ to have a better outcome than FBC. This has led to the conclusion that public awareness of the disease and screening may help in diagnosing this disease at an earlier age and a lower stage and hence lead to improved survival rates. (Miao et al., 2011; Kaushik et al., 2014)

In conclusion, the main purpose of our study was to analyze the data available on MBC in a Pakistani hospital and compare it to the already existing excellent but limited data from both Asian as well as Western studies. We concluded that MBC may have a slow increase in incidence but is still considered a rare disease globally. Screening may help in detection of early disease but is not considered routine due to the rarity of the disease. Public awareness may also help in people seeking medical attention at an early stage leading to improved survival. Tumor stage and lymph node status are the most important prognostic factors for survival. Most importantly, prospective trials are needed to help define guidelines for the management of MBC. Trials, however, may be difficult to conduct given how rare the disease is. Till then, the management of MBC will be based on the management of FBC in both Asian and Western institutes.

Limitations of our study include a retrospective study with a small sample size. However, given the rarity of this disease, only 18 patients were seen at AKUH over 24 years. Chemotherapy details were not available in the patient medical records and therefore could not be analyzed. Statistical analysis was done on a small sample and therefore further research from other Pakistani institutes is needed to enhance the credibility of our study. Our research, however, does shed light on the difference in $\mathrm{MBC}$ in patients from developed and developing countries.

\section{References}

Anderson WF, Jatoi I, Tse J, et al (2010). Male breast cancer: a population-based comparison with female breast cancer. $J$ Clin Oncol, 28, 232-9.

Brinton LA, Richesson DA, Gierach GL, et al (2008). Prospective evaluation of risk factors for male breast cancer. J Natl Cancer Inst, 100, 1477-81.

Chikaraddi SB, Krishnappa R, Deshmane V (2012). Male breast cancer in Indian patients: is it the same? Indian J Cancer, 49, 272-6.

Giordano SH, Cohen DS, Buzdar AU, et al (2004). Breast carcinoma in men: a population-based study. Cancer, 101, 51-7.

Jamal S, Mamoon N, Mushtaq S, et al (2006). Carcinoma of the male breast: a study of 141 cases from northern Pakistan. Asian Pac J Cancer Prev, 7, 119-21.

Jamal S, Mushtaq H, Mubarik A, et al (2009). Estrogen receptor, progesterone receptor, HER2/neu, P53 and Ki-67 status of male breast carcinomas in Pakistan. Asian Pac J Cancer Prev, 10, 1067-70.

Kassim-Lakha S, Bennett J (2013). Philanthropic funding for health in Pakistan. Lancet, 381, 2236-7.

Kaushik M, Oliveira-Cunha M, Shokuhi S (2014). Male breast cancer: a single centre experience and current evidence.
Breast J, 20, 674-5.

Miao H, Verkooijen HM, Chia KS, et al (2011). Incidence and outcome of male breast cancer: an international populationbased study. J Clin Oncol, 29, 4381-6.

Nahleh ZA, Srikantiah R, Safa M, et al (2007). Male breast cancer in the veterans affairs population: a comparative analysis. Cancer, 109, 1471-7.

Sedighi A, Hamed EA, Mohammadian K, et al (2013). Clinicopathologic characteristics of male breast cancer: a report of 21 cases in radiotherapy center of Hamedan, Iran. Asian Pac J Cancer Prev, 14, 7381-3.

Selcukbiricik F, Tural D, Aydogan F, et al (2013). Male breast cancer: 37 -year data study at a single experience center in Turkey. J Breast Cancer, 16, 60-5.

Shah S, Bhattacharyya S, Gupta A, et al (2012). Male breast cancer: a clinicopathologic study of 42 patients in eastern India. Indian J Surg Oncol, 3, 245-9.

Sipetic-Grujicic SB, Murtezani ZH, Neskovic-Konstatinovic $\mathrm{ZB}$, et al (2014). Multivariate analysis of prognostic factors in male breast cancer in Serbia. Asian Pac J Cancer Prev, 15, 3233-8.

Soliman AA, Denewer AT, El-Sadda W, et al (2014). A retrospective analysis of survival and prognostic factors of male breast cancer from a single center. BMC Cancer, 14, 227.

Tasneem S, Khan MM, Khawaja MY (2011). Demographic pattern of male breast cancer: an institutional based study. $J$ Ayub Med Coll Abbottabad, 23, 3-4.

Zhou R, Yu L, Zhou S, et al (2014). Male breast carcinoma: a clinicopathological and immunohistochemical characterization study. Int J Clin Exp Pathol, 7, 6852-61.

Zygogianni AG, Kyrgias G, Gennatas C, et al (2012). Male breast carcinoma: epidemiology, risk factors and current therapeutic approaches. Asian Pac J Cancer Prev, 13, 15-9. 